\title{
KJIM
}

\section{Comparison of the efficacy and safety of direct-acting antiviral therapy with or without hepatitis C-related hepatocellular carcinoma}

\author{
Byung Soo Kwan ${ }^{1}$, Jeong Han Kim ${ }^{1,2}$, Seong Jun Park ${ }^{1}$, Won Hyeok Choe ${ }^{1}$, So Young Kwon ${ }^{1}$, and \\ Byung-Chul Yoo'
}

\author{
${ }^{1}$ Department of Internal Medicine, \\ ${ }^{2}$ Research Institute of Medical \\ Science, Konkuk University School of \\ Medicine, Seoul, Korea
}

Received: September 3, 2019 Revised : October 24, 2019 Accepted: November 24, 2019

\section{Correspondence to}

Jeong Han Kim, M.D.

Department of Internal

Medicine, Konkuk University

School of Medicine, 120

Neungdong-ro, Gwangjin-gu,

Seoul 05029, Korea

Tel: +82-2-2030-7764

Fax: $+82-2-2030-5029$

E-mail:93haan@hanmail.net https://orcid.org/0000-0002$8383-8524$
Background/Aims: Chronic hepatitis C (CHC) treatment has dramatically improved since direct-acting antiviral (DAA) therapy was introduced. However, the use of DAA therapy in CHC patients with hepatocellular carcinoma (HCC) remains controversial. We investigated the DAA treatment response in $\mathrm{CHC}$ patients with HCC.

Methods: We retrospectively analyzed CHC patients treated with DAA from 2016 to 2018. Patients were divided into two groups based on their HCC-history before DAA therapy. Baseline characteristics, sustained virologic response at 12 weeks (SVR 12), and HCC recurrence after DAA therapy were evaluated. We also used propensity score matching (PSM) in a 2:1 ratio to reduce confounding variables.

Results: A total of 192 patients were enrolled; 78.1\% were treatment-naïve, and $34.9 \%$ had liver cirrhosis (LC). Among these patients, 168 did not have HCC, and 24 had HCC. The HCC group was older (57.0 years vs. 72.0 years, $p<0.001$ ), had a higher incidence of LC (26.2\% vs. 95.8\%, $p<0.001$ ), fibrosis- 4 index (2.6 vs. 9.2, $p<$ 0.001 ), liver stiffness measurement (7.0 kPa vs. $17.4 \mathrm{kPa}, p=0.012)$, and $\alpha$-fetoprotein $(4.4 \mathrm{ng} / \mathrm{mL}$ vs. $8.2 \mathrm{ng} / \mathrm{mL}, p \leq 0.001)$. The SVR 12 rate was $97.0 \%$ in the nonHCC group and $91.7 \%$ in the HCC group $(p=0.213)$. HCC recurrence was observed in 14 patients $(58.3 \%)$ in the HCC group.

Conclusions: DAA treatment efficacy in CHC patients with or those without HCC were not significantly different, and HCC recurrence was relatively common.

Keywords: Chronic hepatitis C; Hepatitis C virus; Hepatocellular carcinoma; Direct-acting antiviral

\section{INTRODUCTION}

Hepatitis C virus (HCV) infection is a major cause of chronic liver disease worldwide. In addition, chronic hepatitis $\mathrm{C}(\mathrm{CHC})$ is associated with liver cirrhosis $(\mathrm{LC})$ and hepatocellular carcinoma (HCC) [1]. Globally, HCC is the fifth most common cancer, and death from HCC ranks second among cancer-related deaths [2]. There- fore, patients with hepatitis $\mathrm{C}$ are recommended $\mathrm{HCV}$ treatment to prevent LC and further HCC progression.

In the past, the primary HCV treatment was interferon (IFN) therapy, which had poor efficacy and several limitations. In particular, this therapeutic strategy could be used to treat only a subset of patients due to IFN contraindications, such as advanced LC. As direct-acting antiviral (DAA) therapy has become more common, 
treatment efficacy has increased dramatically, and even patients who were difficult to treat in the past can now be successfully treated [3-5]. However, the use of DAA therapy in CHC patients with HCC is still controversial [6]. Previously, a study using data from the National Veterans Affairs health care system in the United States indicated that the treatment response rate of $\mathrm{CHC}$ patients with HCC was lower than that of $\mathrm{CHC}$ patients without HCC, but the reported response rate in that study may differ from the actual treatment response rate in Korea. In particular, the enrolled patients in that study had type 1 and type 3 genotypes, while patients in Korea predominantly have type 1 and 2 genotypes $[7,8]$.

Another important issue related to the use of DAA therapy for hepatitis C patients with HCC is HCC occurrence and recurrence. Two previous retrospective studies have hypothesized that DAA therapy may be associated with HCC occurrence and recurrence $[9,10]$. Although other studies have raised objections to these studies, the benefits of using DAA as a treatment strategy for CHC patients with HCC remain unclear [11-13].

Therefore, we investigated patient responses to DAA therapy and its safety in CHC patients with or without HCC, as well as the HCC recurrence and HCC occurrence after DAA treatment.

\section{METHODS}

\section{Study design and setting}

In this single-center retrospective cohort study, data on patients who were diagnosed with CHC and started DAA therapy between January 1, 2016, and December 31, 2018, at Konkuk University Medical Center were collected. All enrolled patients were observed for at least 6 months after DAA therapy up to July 31, 2019.

To evaluate the DAA treatment response in CHC patients with or without HCC, patients were divided into two groups: those with HCV-related HCC who achieved that radiologically no viable portion of HCC before DAA therapy and those without HCC before DAA therapy. The patients with no viable portion of HCC, confirmed radiologically, were examined using computed tomography with contrast agent or magnetic resonance imaging with gadoxetic acid (Gd-EOB-DTPA) after treatment to evaluate features such as surgical resection, local ab- lative therapy, and transarterial chemoembolization. Sustained virologic response at 12 weeks (SVR 12) was defined as undetectable hepatitis $\mathrm{C}$ virus ribonucleic acid (HCV-RNA) levels after DAA therapy at 12 weeks. We compared the SVR 12 rate between these two groups.

For subgroup analysis, the HCC group was divided into the HCC recurrence group after DAA therapy and the HCC recurrence-free group. These groups were compared for the associated recurrence risk factor investigation. To analyze HCC occurrence after DAA therapy, patients without HCC before DAA therapy were divided based on HCC occurrence versus HCC-free status after DAA therapy. These groups were compared for related occurrence factor investigations.

The exclusion criteria included patients who were not followed up, those not tested for HCV-RNA at 12 weeks and those who did not complete DAA therapy. This study was approved by the Institutional Review Board of Konkuk University Medical Center (KUH 2019-07-26). Written informed consent by the patients was waived due to a retrospective nature of our study.

\section{Data collection}

We collected patient demographics and clinical variables using electronic medical records, including age, sex, body mass index (BMI), DAA regimen, IFN treatment experience, $\alpha$-fetoprotein (AFP) level, Protein Induced by Vitamin K Absence or Antagonist-II (PIVKA II), serum HCV-RNA level, HCV genotype, Child-Turcotte-Pugh (CTP) score, Model for End-stage Liver Disease (MELD) score, and history of comorbidities. Alcohol drinking was judged to be more than $30 \mathrm{~g}$ daily for men and $20 \mathrm{~g}$ daily for women. The presence of LC was determined by clinical judgment and imaging studies. Additionally, the liver stiffness measurement (LSM) and fibrosis-4 index (FIB-4) were investigated to further examine the presence of LC. LSM was performed using transient elastography. The FIB-4 index was calculated as follows.

FIB-4 index: age (years) $\times$ AST (U/L) / [platelet $\left(10^{9} / \mathrm{L}\right) \times$ alanine aminotransferase $\left.\mathrm{e}^{\mathrm{1} / 2}(\mathrm{U} / \mathrm{L})\right]$

The HCC-related staging systems referenced in this work were the widely recommended Barcelona Clinic Liver Cancer (BCLC) staging [14,15] and modified Union for International Cancer Control (mUICC) staging [15]. 


\section{Statistical analysis}

We used descriptive statistics to describe the baseline demographics of the patients. To reduce the confounding variables, we used propensity score matching (PSM) in a 2:1 ratio to match patients who have HCV-related HCC and non-HCV-related HCC. The PSM was performed including age, sex, and LC. PSM variables such as LC and age were selected due to their association with HCC. The analyzed data were expressed as medians (Q1, Q3) for continuous variables and numbers with percentages for categorical variables. To compare the two groups, we analyzed using Pearson's chi-square test or Fisher's exact test for categorical variables and Mann-Whitney $U$ test for continuous variables. Associated factors of HCC recurrence or occurrence were analyzed using a Firth logistic regression model. Multivariate analysis was performed with variables having a $p<0.05$ in univariate analysis. All $p<0.05$ were considered statistically significant. All statistical analyzes were performed using SAS version 9.4 (SAS Institute Inc., Cary, NC, USA).

\section{RESULTS}

\section{Characteristics of patients with or without HCC be- fore DAA therapy}

Data from a total of 310 patients were collected, and the data from 192 patients were subsequently analyzed after exclusion based on the exclusion criteria (Fig. 1). A total of $54.2 \%$ of the patients were female. The HCV genotype of $55.7 \%$ of patients was type 1 , that of $42.2 \%$ of patients was type $2 a$, and that of $2.1 \%$ of patients was type 2 genotypes, but the subtype could not be specified. Patients who previously received IFN treatment comprised $21.9 \%$ and those with LC comprised $34.9 \%$ of the analyzed patients. The median follow-up duration of these patients was 704.0 days (IQR, 452.5 to 888.0). The baseline clinical characteristics according to the study group are summarized in Table 1.

Among the 192 patients, 168 and 24 patients were divided into groups based on their HCC-history before DAA therapy. Patients with a history of HCC before DAA therapy were more likely to be older (57.0 years vs. 72.0 years, $p<0.001)$ and had a higher incidence of LC $(26.2 \%$ vs. 95.8\%, $p<0.001)$, higher LSM (7.0 kPa vs. $17.4 \mathrm{kPa}, p=$ 0.012 ), higher FIB-4 (2.6 vs. 9.2, $p<0.001$ ), and higher AFP

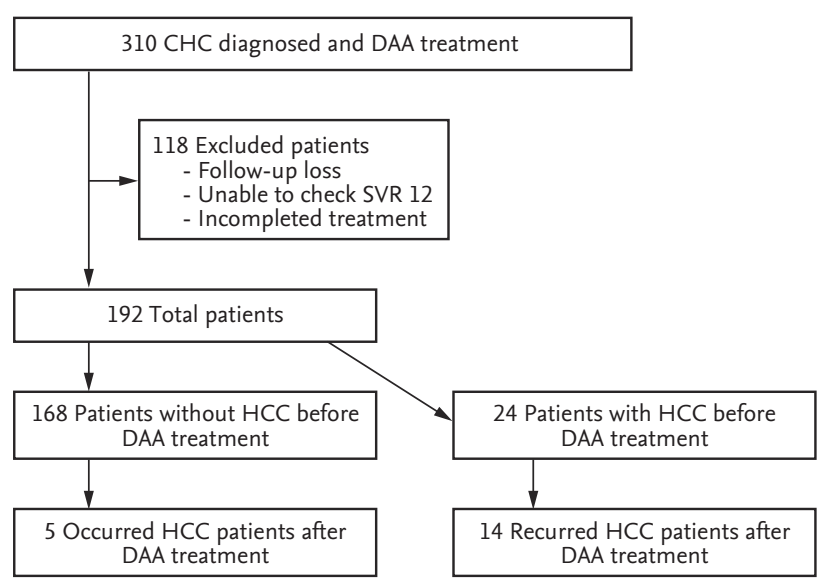

Figure 1. Flow chart of the patient selection process. CHC, chronic hepatitis C; DAA, direct-acting antiviral; SVR 12, sustained virologic response at 12 weeks; HCC, hepatocellular carcinoma.

(4.4 ng/mL vs. $8.2 \mathrm{ng} / \mathrm{mL}, \mathrm{p}<0.001)$. In addition, the DAA regimens used in HCV genotypes 1 and 2 were different between the two groups. In genotype 1, daclatasuvir (DCV) + asunaprevir (ASV) (31.6\%) was the most common in the non-HCC group, and sofosbuvir (SOF) + ledipasvir $(\mathrm{LDV})+$ ribavirin (RBV) (16.7\%) and DCV + ASV (16.7\%) were the most common in the HCC group $(p=0.001)$. In genotype 2, SOF + RBV was the most common in both groups and was more commonly used in the non-HCC group ( $43.5 \%$ vs. $29.2 \%, p=0.018$ ). The SVR 12 rate was $97.0 \%$ in the non-HCC-history group and $91.7 \%$ in the HCC-history group. There was no significant difference between the two groups ( $p=0.213$ ) (Fig. 2 ).

The PSM results for the baseline characteristics are presented in Table 2. As a result of 2:1 matching, 48 and 24 patients were divided into two groups based on their HCC-history before DAA therapy. Patients with HCC before DAA therapy had a higher incidence of LC (37.5\% vs. 95.8\%, $p<0.001$ ), higher FIB-4 (3.9 vs. 9.2, $p<0.001$ ), and higher AFP (4.6 ng/mL vs. $8.2 \mathrm{ng} / \mathrm{mL}, p<0.001$ ). DAA regimens used in HCV genotype 1 were different between the two groups. DCV + ASV (33.3\%) was the most common regimen in the non-HCC group, whereas $\mathrm{SOF}+\mathrm{LDV}+$ RBV (16.7\%) and DCV + ASV (16.7\%) were the most common in the HCC group ( $p=0.025)$. In the HCC group, 46 patients $(63.9 \%)$ were genotype 1 and had a higher incidence of LC (40.0\% vs. 93.8\%, p = 0.001) (Supplementary Table 1); 26 patients (36.1\%) were genotype 2 and had a 
Kwan BS, et al. DAA therapy in hepatitis C with HCC

Table 1. Baseline clinical characteristics of patients with or without HCC before DAA treatment

\begin{tabular}{|c|c|c|c|c|}
\hline Variable & Total $(\mathrm{n}=192)$ & Without HCC $(n=168)$ & With HCC $(n=24)$ & $p$ value \\
\hline Age, yr & $59.0(52.0-67.0)$ & $57.0(51.0-64.0)$ & $72.0(66.5-75.5)$ & $<0.001$ \\
\hline Female sex & $104(54.2)$ & $95(56.5)$ & $9(37.5)$ & 0.125 \\
\hline $\mathrm{BMI}, \mathrm{kg} / \mathrm{m}^{2}$ & $23.6(21.6-26.0)$ & $23.4(21.1-25.5)$ & $24.6(22.8-28.2)$ & 0.084 \\
\hline HCV-RNA, IU/mL & $\begin{array}{c}1,510,000.0 \\
(289,000.0-4,580,000.0)\end{array}$ & $\begin{array}{c}1,575,000.0 \\
(307,500.0-5,135,000.0)\end{array}$ & $\begin{array}{c}2,841,716.7 \\
(166,800.0-3,855,000.0)\end{array}$ & 0.612 \\
\hline Genotype & & & & 0.351 \\
\hline 1 & $107(55.7)$ & $91(54.2)$ & $16(66.7)$ & \\
\hline 2 & $85(44.3)$ & $77(45.8)$ & $8(33 \cdot 3)$ & \\
\hline DAA for genotype 1 & & & & 0.001 \\
\hline $\mathrm{DCV}+\mathrm{ASV}$ & $57(29.7)$ & $53(31.6)$ & $4(16.7)$ & \\
\hline $\mathrm{EBR}+\mathrm{GZR}$ & $22(11.5)$ & $19(11.3)$ & $3(12.5)$ & \\
\hline $\mathrm{OBV} / \mathrm{PTV} / \mathrm{r}+\mathrm{DSV}$ & $13(6.8)$ & $11(6.5)$ & $2(8.3)$ & \\
\hline $\mathrm{SOF}+\mathrm{LDV}$ & $10(5.2)$ & $7(4.2)$ & $3(12.5)$ & \\
\hline $\mathrm{SOF}+\mathrm{LDV}+\mathrm{RBV}$ & $5(2.6)$ & $1(0.6)$ & $4(16.7)$ & \\
\hline DAA for genotype 2 & & & & 0.018 \\
\hline $\mathrm{SOF}+\mathrm{DCV}$ & $1(0.5)$ & o & $1(4.2)$ & \\
\hline $\mathrm{SOF}+\mathrm{LDV}+\mathrm{RBV}$ & $2(1.0)$ & $2(1.2)$ & o & \\
\hline $\mathrm{SOF}+\mathrm{RBV}$ & $80(41.7)$ & $73(43.5)$ & $7(29.2)$ & \\
\hline GLE + PIB & $2(1.0)$ & $2(1.2)$ & o & \\
\hline SVR 12 & $185(96.4)$ & $163(97.0)$ & $22(91.7)$ & 0.213 \\
\hline Prior IFN experienced & $42(21.9)$ & $33(19.6)$ & $9(37.5)$ & 0.086 \\
\hline Liver cirrhosis & $67(34.9)$ & $44(26.2)$ & $23(95.8)$ & $<0.001$ \\
\hline $\mathrm{LSM}, \mathrm{kPa}^{\mathrm{a}}$ & $7.4(4.4-12.6)$ & $7.0(4.4-11.5)$ & $17.4(14.0-48.0)$ & 0.012 \\
\hline FIB-4 & $3.0(1.8-4.7)$ & $2.6(1.6-4.1)$ & $9.2(4.7-11.4)$ & $<0.001$ \\
\hline$>3.25$ & $85(44.7)$ & $62(37.3)$ & $23(95.8)$ & $<0.001$ \\
\hline CTP score & & & & 0.064 \\
\hline A & $163(84.9)$ & $143(94.7)$ & $20(83.3)$ & \\
\hline B & $12(6.3)$ & $8(5 \cdot 3)$ & $4(16.7)$ & \\
\hline MELD score & & & & 0.182 \\
\hline$<9$ & $123(73.7)$ & $110(75.9)$ & $13(59.1)$ & \\
\hline $10-19$ & $40(24.0)$ & $32(22.1)$ & $8(36.4)$ & \\
\hline $20-29$ & $4(2.4)$ & $3(2.1)$ & $1(4.6)$ & \\
\hline AFP, ng/mL & $4.9(3.0-9.2)$ & $4.4(2.8-8.6)$ & $8.2(6.2-31.1)$ & $<0.001$ \\
\hline Alcohol & $41(21.4)$ & $37(22.0)$ & $4(16.7)$ & 0.739 \\
\hline Side effect & $68(35.4)$ & $60(35.7)$ & $8(33 \cdot 3)$ & 1.000 \\
\hline
\end{tabular}

Values are presented as median (interquartile range) or number (\%).

HCC, hepatocellular carcinoma; DAA, direct-acting antiviral; BMI, body mass index; HCV-RNA, hepatitis C virus ribonucleic acid; DCV, daclatasuvir; ASV, asunaprevir; EBR, elbasvir; GZR, grazoprevir; OBV, ombitasvir; PTV, paritaprevir; r, ritonavir; DSV, dasabuvir; SOF, sofosbuvir; LDV, ledipasvir; RBV, ribavirin; GLE, glecaprevir; PIB, pibrentasvir; SVR 12, sustained viral response at 12 weeks; IFN, interferon; LSM, liver stiffness measurement; FIB-4, fibrosis-4; CTP, Child-Turcotte-Pugh; MELD, model for End-stage Liver Disease; AFP, $\alpha$-fetoprotein.

${ }^{\mathrm{a}} \mathrm{A}$ total of 90 patients checked LSM, 86 in the without HCC group and 4 in the with HCC group. 

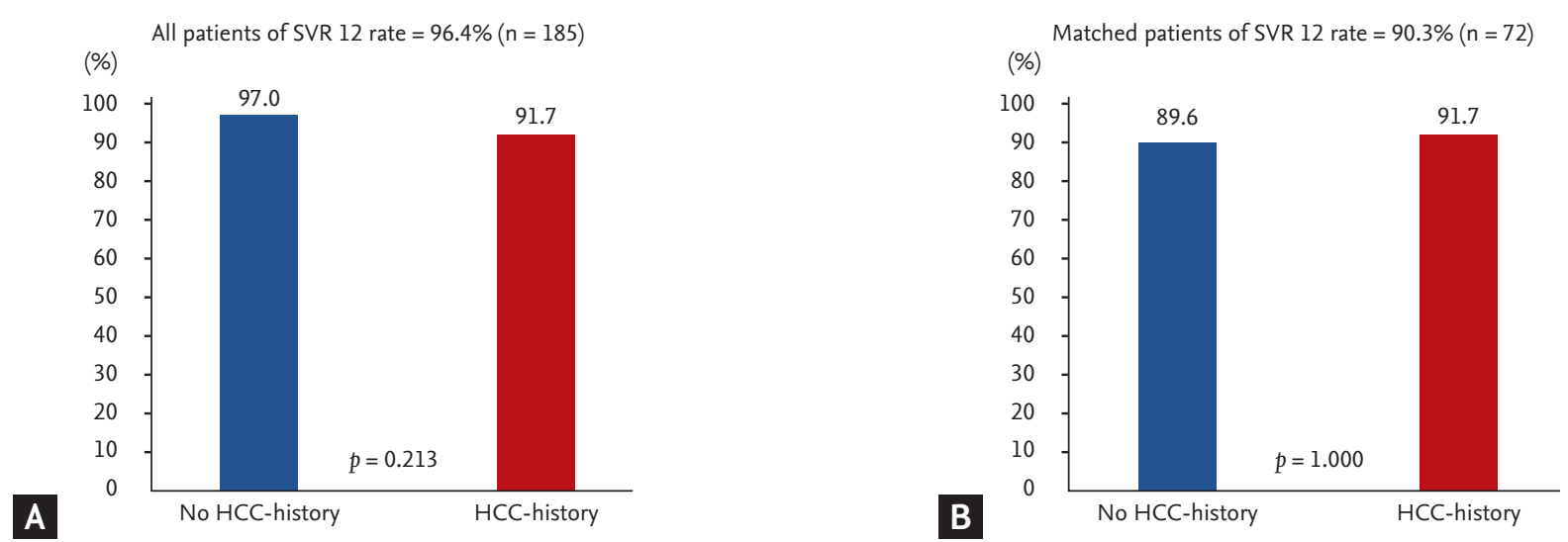

Figure 2. Sustained virologic response at 12 weeks (SVR 12) rates of all patients and matched patients after direct-acting antiviral (DAA) therapy. (A) Among the 192 patients, SVR 12 was achieved in 185 (96.4\%). The SVR 12 rate was $97.0 \%$ in the without hepatocellular carcinoma (HCC)-history group and $91.7 \%$ in the HCC-history group $(p=0.213)$. (B) Among the 72 matched patients, the SVR 12 rate was $90.3 \%$. The SVR 12 rate was $89.6 \%$ in the without HCC-history group and $91.7 \%$ in the HCC-history group $(p=1.000)$.

higher incidence of LC (33.3\% vs. 100\%, $p=0.002)$ (Supplementary Table 2 ) and a relatively higher model for end-stage liver disease with incorporation of serum sodium (MELD-NA) score $(p=0.026)$ (Supplementary Table 2). The SVR 12 rate was $89.6 \%$ in the non-HCC group and $91.7 \%$ in the HCC group, though there was no significant difference between the two groups $(p=1.000)$ (Fig. 2 ).

\section{Adverse event of HCC-history patients after DAA therapy}

Adverse events of DAA therapy are described in Table 3. Eight patients (33.3\%) developed adverse events in the HCC group, of which three patients were genotype 1 and five patients were 2 . The most common adverse event in the HCC group was anemia, which was defined as hemoglobin $<10 \mathrm{~g} / \mathrm{dL}$ or decreased hemoglobin $>2 \mathrm{~g} / \mathrm{dL}$ from baseline hemoglobin ( $\mathrm{n}=4,16.7 \%)$. In addition, a tingling sensation $(\mathrm{n}=2,8.3 \%)$, insomnia $(\mathrm{n}=1,4.2 \%)$, and minor adverse events ( $\mathrm{n}=1,4.2 \%)$, occurred. There were no severe adverse events in either group that would lead to discontinuation of DAA therapy. Additionally, there was no significant difference in the number of adverse events between the groups regardless of the presence of HCC (35.7\% vs. $33.3 \%, p=0.176)$. Similarly, PSM results showed no difference in adverse events between the groups $(p=0.858)$ (Table 2$)$.

\section{Comparison according to HCC recurrence in patients with a history of $\mathrm{HCC}$ and associated factors}

Fourteen of 24 patients (58.3\%) showed cumulative HCC recurrence. The median follow-up duration of the HCC group was 709.5 days (interquartile range [IQR], 539.0 to 815.5), and the total median period after DAA therapy to recurrence was 92.0 days (IQR, 31.o to 122.0). Specifically, the median period after DAA therapy to recurrence was 92.0 days (IQR, 31.0 to 103.0) in the curative treated HCC group and 101.0 days (IQR, 88.0 to 483.0) in the palliative treated HCC group, and no significant difference was found between the two groups $(p=0.422)$. Patients with HCC-history before DAA therapy were divided according to the presence of HCC recurrence. A higher number of patients in the HCC recurrence group had a previous experience with IFN treatment ( $0 \%$ vs. $64.3 \%, p=$ 0.002) than in the non-recurrence group (Table 4). In the multivariable logistic regression analysis, a statistically significant factor was found for previous IFN treatment (odds ratio [OR], 36.26; 95\% confidence interval [CI], 1.51 to $872.74 ; p=0.027$ ) (Table 5).

\section{Comparison of $\mathrm{HCC}$ occurrence in patients without a history of $\mathrm{HCC}$ and associated factors}

Five patients were found to have HCC after DAA therapy (3.0\%). The time for the median follow-up duration of the non-HCC group was 699.0 days (IQR, 427.5 to 898.5), and the median period after DAA therapy to occurrence was 110.0 days (IQR, 98.0 to 217.0). The HCC occurrence 
Kwan BS, et al. DAA therapy in hepatitis $\mathrm{C}$ with $\mathrm{HCC}$

Table 2. Propensity score matching results of baseline clinical characteristics

\begin{tabular}{|c|c|c|c|c|}
\hline Variable & $\operatorname{Total}(\mathrm{n}=72)$ & Without HCC $(\mathrm{n}=48)$ & With HCC $(n=24)$ & $p$ value \\
\hline Age, yr & $71.0(63.0-76.5)$ & $70.8(63.0-77.5)$ & $71.0(66.5-75.5)$ & 0.886 \\
\hline Female sex & $24(33 \cdot 3)$ & $15(31.3)$ & $9(37.5)$ & 0.596 \\
\hline $\mathrm{BMI}, \mathrm{kg} / \mathrm{m}^{2}$ & $24.1(22.4-26.0)$ & $23.6(22.0-25.4)$ & $25.1(22.7-28.3)$ & 0.142 \\
\hline HCV-RNA, IU/mL & $\begin{array}{c}980,000.0 \\
(96,800.0-4,400,000.0)\end{array}$ & $\begin{array}{c}3,367,365.0 \\
(92,200.0-5,180,000.0)\end{array}$ & $\begin{array}{c}2,841,716.7 \\
(166,800.0-3,855,000.0)\end{array}$ & 0.693 \\
\hline Genotype & & & & 0.729 \\
\hline 1 & $46(63.9)$ & $30(62.5)$ & $16(66.7)$ & \\
\hline 2 & $26(36.1)$ & $18(37.5)$ & $8(33 \cdot 3)$ & \\
\hline DAA for genotype 1 & & & & 0.025 \\
\hline $\mathrm{DCV}+\mathrm{ASV}$ & $20(27.8)$ & $16(33.3)$ & $4(16.7)$ & \\
\hline $\mathrm{EBR}+\mathrm{GZR}$ & $11(15 \cdot 3)$ & $8(16.7)$ & $3(12.5)$ & \\
\hline $\mathrm{OBV} / \mathrm{PTV} / \mathrm{r}+\mathrm{DSV}$ & $6(8.3)$ & $4(8.3)$ & $2(8.3)$ & \\
\hline $\mathrm{SOF}+\mathrm{LDV}$ & $5(6.9)$ & $2(4.2)$ & $3(12.5)$ & \\
\hline $\mathrm{SOF}+\mathrm{LDV}+\mathrm{RBV}$ & $4(5.6)$ & o & $4(16.7)$ & \\
\hline DAA for genotype 2 & & & & 0.256 \\
\hline $\mathrm{SOF}+\mathrm{DCV}$ & $1(1.4)$ & o & $1(4.2)$ & \\
\hline $\mathrm{SOF}+\mathrm{LDV}+\mathrm{RBV}$ & $1(1.4)$ & $1(2.1)$ & o & \\
\hline $\mathrm{SOF}+\mathrm{RBV}$ & $24(33 \cdot 3)$ & $17(35.4)$ & $7(29.2)$ & \\
\hline SVR 12 & $65(90.3)$ & $43(89.6)$ & $22(91.7)$ & 1.000 \\
\hline Prior IFN experienced & $21(29.2)$ & $12(25.0)$ & $9(37.5)$ & 0.271 \\
\hline Liver cirrhosis & $41(56.9)$ & $18(37.5)$ & $23(95.8)$ & $<0.001$ \\
\hline $\mathrm{LSM}, \mathrm{kPa}^{\mathrm{a}}$ & $7.7(5.2-14.0)$ & $6.7(4.4-11.8)$ & $17.4(14.0-48.0)$ & 0.024 \\
\hline FIB-4 & $4.4(3.1-10.2)$ & $3.9(2.3-5.3)$ & $9.2(4.7-11.4)$ & $<0.001$ \\
\hline$>3.25$ & $52(73.2)$ & $29(61.7)$ & $23(95.8)$ & 0.005 \\
\hline CTP score & & & & 0.715 \\
\hline A & $56(86.2)$ & $36(87.8)$ & $20(83.3)$ & \\
\hline B & $9(13.8)$ & $5(12.2)$ & $4(16.7)$ & \\
\hline MELD score & & & & 1.000 \\
\hline$<9$ & $38(61.3)$ & $25(62.5)$ & $13(59.1)$ & \\
\hline $10-19$ & $22(35.5)$ & $14(35.0)$ & $8(36.4)$ & \\
\hline $20-29$ & $2(3.2)$ & $1(2.5)$ & $1(4 \cdot 6)$ & \\
\hline AFP, ng/mL & $7.1(3.3-14.0)$ & $4.6(2.8-10.8)$ & $8.2(6.2-31.1)$ & 0.012 \\
\hline Alcohol & $17(23.6)$ & $13(27.1)$ & $4(16.7)$ & 0.492 \\
\hline Side effect & $23(31.9)$ & $15(31.3)$ & $8(33.33)$ & 0.858 \\
\hline
\end{tabular}

Values are presented as median (interquartile range) or number (\%).

HCC, hepatocellular carcinoma; BMI, body mass index; HCV-RNA, hepatitis C virus ribonucleic acid; DAA, direct-acting antiviral; DCV, daclatasuvir; ASV, asunaprevir; EBR, elbasvir; GZR, grazoprevir; OBV, ombitasvir; PTV, paritaprevir; r, ritonavir; DSV, dasabuvir; SOF, sofosbuvir; LDV, ledipasvir; RBV, ribavirin; SVR 12, sustained viral response at 12 weeks; IFN, interferon; LSM, liver stiffness measurement; FIB-4, fibrosis-4; CTP, Child-Turcotte-Pugh; MELD, Model for End-stage Liver Disease; AFP, $\alpha$-fetoprotein.

${ }^{a} A$ total of 22 patients checked LSM, 18 in the without HCC group and 4 in the with HCC group. 
Table 3. Adverse event of direct-acting antiviral treatment

\begin{tabular}{lcll}
\hline Variable & $\begin{array}{c}\text { Without HCC } \\
(\mathrm{n}=168)\end{array}$ & $\begin{array}{c}\text { With HCC } \\
(\mathrm{n}=24)\end{array}$ & p value \\
\hline Total patients & $60(35.7)$ & $8(33.3)$ & 0.176 \\
$\quad$ Genotype 1 & $19(11.3)$ & $3(12.5)$ & \\
\multicolumn{1}{c}{ Genotype 2 } & $41(24.4)$ & $5(20.8)$ & \\
Discontinuation of & 0 & 0 & \\
treatment & & & \\
Anemia & & & \\
Dyspepsia & $35(20.8)$ & $4(16.7)$ & \\
Insomnia & $5(3.0)$ & $0(0.0)$ & \\
Fatigue & $4(2.4)$ & $1(4.2)$ & \\
Tingling sensation & 0 & 0 \\
Arrhythmic events & $2(1.2)$ & 0 & \\
Cough & $1(0.6)$ & 0 & \\
Minority events & $6(3.6)^{\mathrm{b}}$ & $1(4.2)^{\mathrm{c}}$ & \\
\hline
\end{tabular}

Values are presented as number (\%).

HCC, hepatocellular carcinoma.

${ }^{\mathrm{a}}$ Hemoglobin $<10 \mathrm{~g} / \mathrm{dL}$ or decreased hemoglobin $>2 \mathrm{~g} / \mathrm{dL}$ from baseline.

${ }^{b}$ Dizziness (1), headache (1), skin rash (1), xeroderma (1), edema (1), epistaxis (1).

${ }^{\mathrm{c}} \operatorname{Edema}(1)$.

group was relatively older $(57.0$ years vs. 64.0 years, $p=$ $0.015)$ and had a higher BMI $\left(23.3 \mathrm{~kg} / \mathrm{m}^{2}\right.$ vs. $25.9 \mathrm{~kg} / \mathrm{m}^{2}$, $p=0.027$ ), higher FIB-4 levels (2.5 vs. 5.9, $p=0.009$ ), and higher AFP (4.3 ng/mL vs. $22.9 \mathrm{ng} / \mathrm{mL}, p=0.002$ ) than the non-occurrence group. Moreover, the SVR 12 rate of the HCC occurrence group was lower than that the nonHCC occurrence group ( $98.2 \%$ vs. $60.0 \%, p=0.007$ ), and the proportion of LC in the HCC occurrence group was relatively high (26.0\% vs. $60.0 \%, p=0.113$ ) (Table 6$)$. Of the 44 patients with LC, three had HCC, and two of 119 without LC had HCC (6.8\% vs. $1.6 \%, p=0.219)$.

PSM results regarding HCC occurrence in without HCC-history patients are presented in Table 6. The HCC occurrence group had a higher BMI $\left(23.4 \mathrm{~kg} / \mathrm{m}^{2}\right.$ vs. $\left.25.9 \mathrm{~kg} / \mathrm{m}^{2}, p=0.009\right)$ and higher $\mathrm{AFP}(3.8 \mathrm{ng} / \mathrm{mL}$ vs. 22.9 $\mathrm{ng} / \mathrm{mL}, p=0.006$ ).

Age was identified in multivariate analysis as a factor associated with HCC occurrence (OR, 1.12; 95\% CI, 1.02 to $1.23 ; p=0.021$ ) (Table 5 ).

\section{DISCUSSION}

HCV treatment has become commonplace as DAA therapy has improved the cure rate, and it has become possible to treat patients who had difficulties with IFN treatment in the past. The treatment of HCV patients with a history of HCC is also possible because of DAA therapy. Previous studies have shown that there are several reasons why CHC patients with a history of HCC should be treated. The treatment of CHC in patients with decompensated LC can improve their LC and improve liver function, which can have positive effects [16]. In addition, referring to the literature written in the interferon era, it has been confirmed that hepatitis $\mathrm{C}$ treatment can ultimately reduce HCC recurrence [17-19].

Beste et al. [7] found that CHC patients with a history of HCC had lower SVR 12 rates than those without HCC-history (74.4\% vs. 91.1\%). However, in our study, these groups showed no significant difference in the SVR 12 rate (overall, 91.7\% vs. 97.0\%, $p=0.213$; PSM, $89.6 \%$ vs. $91.7 \%, p=1.000)$. There are several reasons to explain this difference. First, the enrolled patient populations have different demographic characteristics. Our study enrolled Asian patients with genotypes $1 \mathrm{~b}$ and $2 \mathrm{a}$. However, the previous study included many nonhispanic white patients with genotypes 1 and 3. Second, all patients with cirrhosis in this study showed compensated LC, but in the previous study, $31.5 \%$ of the enrolled patients showed decompensated LC.

HCC recurrence was observed in $58.3 \%$ of patients with HCC. A previous literature review reported HCC recurrence after DAA therapy for CHC with HCC patients, and the cumulative 2-year recurrence rate was 38.9\% to $54.5 \%[20,21]$. Compared with these studies, given that the median duration was 704.0 days (IQR, 452.5 to 888.0) in our study, the HCC recurrence rate of our study was similar (58.3\% vs. $38.9 \%$ to $54.5 \%$ ). Additionally, the time for the median period after DAA therapy to HCC recurrence was 92.0 days (IQR, 31.0 to 122.0), suggesting that patients who experienced recurrence after DAA therapy were identified in a relatively short period of time.

The HCC recurrence-related factor was previous IFN treatment, and multivariable logistic regression analysis of relevant factors showed the same results (OR, 36.26; $95 \%, 1.51$ to $872.74 ; p=0.027$ ). A previous study on the associated factors of HCC recurrence showed that SVR 
Kwan BS, et al. DAA therapy in hepatitis C with HCC

Table 4. Comparison of HCC recurrence in HCC-history patients

\begin{tabular}{|c|c|c|c|}
\hline Variable & No recurrence $(n=10)$ & Recurrence $(n=14)$ & $p$ value \\
\hline Age, yr & $69.0(63.0-73.0)$ & $73.5(71.0-78.0)$ & 0.069 \\
\hline Female sex & $5(50.0)$ & $4(28.6)$ & 0.403 \\
\hline $\mathrm{BMI}, \mathrm{kg} / \mathrm{m}^{2}$ & $23.4(21.6-24.2)$ & $26.3(23.7-28.3)$ & 0.069 \\
\hline HCV-RNA, IU/mL & $1,031,000.0(568,000.0-8,230,000.0)$ & $1,827,500.0(93,600.0-3,560,000.0)$ & 0.837 \\
\hline Genotype & & & 1.000 \\
\hline 1 & $7(70.0)$ & $9(64.3)$ & \\
\hline 2 & $3(30.0)$ & $5(35 \cdot 7)$ & \\
\hline DAA for genotype 1 & & & 0.126 \\
\hline $\mathrm{DCV}+\mathrm{ASV}$ & o & $4(44 \cdot 4)$ & \\
\hline $\mathrm{EBR}+\mathrm{GZR}$ & $3(42.9)$ & o & \\
\hline $\mathrm{OBV} / \mathrm{PTV} / \mathrm{r}+\mathrm{DSV}$ & $1(14 \cdot 3)$ & $1(11.1)$ & \\
\hline $\mathrm{SOF}+\mathrm{LDV}$ & $1(14.3)$ & $2(22.2)$ & \\
\hline $\mathrm{SOF}+\mathrm{LDV}+\mathrm{RBV}$ & $2(28.6)$ & $2(22.2)$ & \\
\hline DAA for genotype 2 & & & 0.783 \\
\hline $\mathrm{SOF}+\mathrm{DCV}$ & $1(33.3)$ & o & \\
\hline $\mathrm{SOF}+\mathrm{RBV}$ & $2(66.7)$ & $5(100.0)$ & \\
\hline SVR 12 & $10(100.0)$ & $12(85.7)$ & 0.493 \\
\hline Prior IFN experienced & o & $9(64 \cdot 3)$ & 0.002 \\
\hline Liver cirrhosis & $9(90.0)$ & $14(100.0)$ & 0.417 \\
\hline FIB-4 & $4.8(4.5-9.1)$ & $10.8(6.7-15.1)$ & 0.064 \\
\hline$>3.25$ & $10(100.0)$ & $13(92.9)$ & 1.000 \\
\hline CTP score & & & 1.000 \\
\hline A & $8(80.0)$ & $12(85.7)$ & \\
\hline B & $2(20.0)$ & $2(14 \cdot 3)$ & \\
\hline MELD-NA score & & & 0.814 \\
\hline$<9$ & $7(70.0)$ & $6(50.0)$ & \\
\hline $10-19$ & $3(30.0)$ & $5(41.7)$ & \\
\hline $20-29$ & 0 & $1(8.3)$ & \\
\hline AFP before DAA & $9.9(6.2-24.3)$ & $7.7(5.7-37.9)$ & 0.305 \\
\hline PIVKA II before DAA & $14.8(11.0-22.8)$ & $18.0(13.0-19.5)$ & 0.926 \\
\hline HCC treatment & & & 0.780 \\
\hline Curative $^{\mathrm{a}}$ & $5(50.0)$ & $5(35.7)$ & \\
\hline Palliative $^{\mathrm{b}}$ & $5(50.0)$ & $9(64 \cdot 3)$ & \\
\hline BCLC stage & & & 0.629 \\
\hline o state & $3(30.0)$ & $5(35.7)$ & \\
\hline A stage & $7(70.0)$ & $8(57.1)$ & \\
\hline B stage & o & $1(7.1)$ & \\
\hline mUICC stage & & & 0.773 \\
\hline 1 stage & $3(30.0)$ & $5(35.7)$ & \\
\hline 2 stage & $5(50.0)$ & $5(35.7)$ & \\
\hline 3 stage & $2(20.0)$ & $4(28.6)$ & \\
\hline Maximum tumor size, $\mathrm{cm}$ & $2.2(1.4-2.5)$ & $2.0(1.4-2.4)$ & 0.769 \\
\hline
\end{tabular}


Table 4. Contiuned

\begin{tabular}{|c|c|c|c|}
\hline Variable & No recurrence $(\mathrm{n}=10)$ & Recurrence $(\mathrm{n}=14)$ & $p$ value \\
\hline HCC nodules & & & 0.678 \\
\hline 1 & $8(80.0)$ & $10(71.4)$ & \\
\hline 2 & $2(20.0)$ & $3(21.4)$ & \\
\hline 3 & o & $1(7.1)$ & \\
\hline $\begin{array}{l}\text { From last HCC treatment to } \\
\text { DAA treatment, day }\end{array}$ & $188.5(54.0-619.0)$ & $214.0(70.0-645.0)$ & 0.883 \\
\hline
\end{tabular}

Values are presented as median (interquartile range) or number (\%).

HCC, hepatocellular carcinoma; BMI, body mass index; HCV-RNA, hepatitis C virus ribonucleic acid; DAA, direct-acting antiviral; DCV, daclatasuvir; ASV, asunaprevir; EBR, elbasvir; GZR, grazoprevir; OBV, ombitasvir; PTV, paritaprevir; r, ritonavir; DSV, dasabuvir; SOF, sofosbuvir; LDV, ledipasvir; RBV, ribavirin; SVR 12, sustained viral response at 12 weeks; IFN, interferon; FIB-4, fibrosis-4; CTP, Child-Turcotte-Pugh; MELD-NA, model for end-stage liver disease with incorporation of serum sodium; AFP, $\alpha$-fetoprotein; PIVKA II, Protein Induced by Vitamin K Absence or Antagonist-II; BCLC, Barcelona Clinic Liver Cancer; mUICC, modified Union for International Cancer Control.

${ }^{a}$ Liver transplantation or Surgical resection or Local ablation or transarterial chomoembolization plus local ablation.

${ }^{\mathrm{b}}$ Transarterial chemoembolization.

12 rate, time between the last HCC treatment and DAA therapy initiation, noncurative HCC treatment, more than one HCC treatment before DAA therapy, tumor size, LC, and AFP-L3\% were relevant factors $[15,20,22]$. A recent study reported that patients with previous IFN experience have a high risk of de novo HCC, and the same results were found in our study [23]. As a theory supporting this result, it was estimated that this is due to the immunomodulatory and antitumor properties of IFN rather than antiviral activity alone [24]. However, this issue is controversial and should be evaluated by more studies.

In our study, the cumulative rate of HCC occurrence after DAA therapy was $3.0 \%$ in the non-HCC group during the median follow-up duration (699.0 days [IQR, 427.5 to 898.5]). Previous studies have shown that the incidence of HCC after DAA therapy was $4.9 \%$ in all patients for 3 years. In addition, patients with LC had a higher occurrence of HCC than those with non-LC (10.0\% vs. $2.9 \%, p<0.0001)[11]$. When comparing previous studies, patients with LC did not have a statistically higher occurrence of HCC than those without LC (6.8\% vs. 1.6\%, $p=0.219)$, and the HCC occurrence rate in our study was similar (3.0\% vs. $4.9 \%$ ) to that in other studies.

The PSM results revealed that the HCC occurrence group had a higher BMI and higher AFP than the non-occurrence group. It is well known that the risk of HCC increases with AFP level. BMI is confirmed to be an independent factor in previous studies that have been linked to HCC [25].

The SVR 12 rate was lower in the HCC occurrence group than in the non-HCC group (98.2\% vs. $60.0 \%, p=$ 0.007). Although the SVR 12 rate was found to be lower in the HCC group, more investigation is needed to determine whether SVR failure is a risk factor for HCC because a relatively small number of patients were enrolled in our study. Additionally, the proportion of cirrhosis in the HCC occurrence of this study is high, but the lack of significant differences between the two groups is probably due to a lack of sufficient numbers $(p=0.113)$.

The limitations of our study are its relatively small sample size and its single-center retrospective cohort study nature. As a limitation of the small sample size, there were fewer patients with HCC, and thus, the comparisons that can be made are limited. In particular, the difference among DAA regimens could not be evaluated. Moreover, the LC and FIB-4 were not corrected by PSM because the LC and above the 3.25 of FIB- 4 in the HCC group was $95.8 \%$, which is a very high rate that could not be statistically matched in the non-HCC group (LC, $37.5 \%$ vs. $95.8 \%, p<0.001$; FIB- $4>3.25,61.7 \%$ vs. $95.8 \%, p$ $=0.005)$. Despite these limitations, this study is the first report from Korea, and any additional information on the association of DAA therapy with HCC is clinically important and meaningful.

In conclusion, the SVR 12 rate of DAA therapy among 
Table 5. Univariable and multivariable logistic regression analysis of HCC recurrence and HCC occurrence

\begin{tabular}{|c|c|c|c|c|c|c|c|c|}
\hline \multirow{3}{*}{ Variable } & \multicolumn{4}{|c|}{ Recurrence } & \multicolumn{4}{|c|}{ Occurrence } \\
\hline & \multicolumn{2}{|l|}{ Univariate } & \multicolumn{2}{|c|}{ Multivariate } & \multicolumn{2}{|l|}{ Univariate } & \multicolumn{2}{|c|}{ Multivariate } \\
\hline & OR $(95 \% \mathrm{CI})$ & $p$ value & OR $(95 \% \mathrm{CI})$ & $p$ value & OR $(95 \% \mathrm{CI})$ & $p$ value & OR $(95 \% \mathrm{CI})$ & $p$ value \\
\hline Age, yr & $1.13(0.98-1.30)$ & 0.085 & & & $1.10(1.01-1.19)$ & 0.021 & $1.12(1.02-1.23)$ & 0.021 \\
\hline $\begin{array}{l}\text { Sex, male vs. } \\
\text { female }\end{array}$ & $2.50(0.46-13.65)$ & 0.290 & & & $5.45(0.60-49.83)$ & 0.133 & & \\
\hline BMI & $1.48(0.97-2.27)$ & 0.067 & & & $1.34(1.00-1.80)$ & 0.049 & $1.34(0.99-1.82)$ & 0.057 \\
\hline HCV-RNA & $1.00(1.00-1.00)$ & 0.210 & & & $1.00(1.00-1.00)$ & 0.192 & & \\
\hline Genotype, 2 vs. 1 & $1.30(0.23-7.38)$ & 0.770 & & & $0.78(0.13-4.81)$ & 0.791 & & \\
\hline SVR 12 & $4.20(0.09-190.18)$ & 0.461 & & & $35.56(4.26-297.10)$ & 0.001 & $8.12\left(0.55^{-120.84}\right)$ & 0.128 \\
\hline $\begin{array}{l}\text { Prior IFN } \\
\text { experienced }\end{array}$ & $36.26(1.51-872.74)$ & 0.027 & $36.26(1.51-872.74)$ & 0.027 & $0.35(0.02-6.84)$ & 0.492 & & \\
\hline Liver cirrhosis & $4.65(0.05-471.65)$ & 0.515 & & & $4.46(0.72-27.65)$ & 0.108 & & \\
\hline FIB-4 & $1.19(0.97-1.46)$ & 0.100 & & & $1.09(0.96-1.25)$ & 0.199 & & \\
\hline AFP before DAA & $0.99(0.98-1.01)$ & 0.364 & & & $1.02(1.00-1.04)$ & 0.019 & & \\
\hline CTP score A vs. B & $1.50(0.17-12.94)$ & 0.712 & & & $0.55(0.02-13.03)$ & 0.710 & & \\
\hline $\begin{array}{l}\text { HCC treatment, } \\
\text { Curative }^{\mathrm{a}} \text { vs. } \\
\text { palliative }^{\mathrm{b}}\end{array}$ & $0.57(0.11-2.90)$ & 0.486 & & & & & & \\
\hline \multicolumn{9}{|l|}{ MELD-NA score } \\
\hline $10-19$ vs. $<9$ & $1.81(0.30-10.87)$ & 0.983 & & & $1.46(0.20-10.57)$ & 0.773 & & \\
\hline $20-29$ vs. $<9$ & $3.49(0.03-369.44)$ & 0.687 & & & $4.39(0.12-158.16)$ & 0.478 & & \\
\hline \multicolumn{9}{|l|}{ BCLC stage } \\
\hline Stage A vs. o & $0.72(0.13-4.13)$ & 0.621 & & & & & & \\
\hline Stage B vs. o & $1.91(0.02-220.36)$ & 0.730 & & & & & & \\
\hline \multicolumn{9}{|l|}{ mUICC stage } \\
\hline Stage 2 vs. 1 & $0.60(0.10-3.99)$ & 0.478 & & & & & & \\
\hline Stage 3 vs. 1 & $1.20(0.13-11.05)$ & 0.659 & & & & & & \\
\hline $\begin{array}{l}\text { Maximum } \\
\text { tumor size }\end{array}$ & $1.38(0.53-3.56)$ & 0.509 & & & & & & \\
\hline
\end{tabular}

No. of HCC nodules

\begin{tabular}{ccc}
2 vs. 1 & $1.13(0.15-8.46)$ & 0.826 \\
3 vs. 1 & $2.48(0.02-257.21)$ & 0.722 \\
\hline
\end{tabular}

HCC, hepatocellular carcinoma; OR, odds ratio; CI, confidence interval; BMI, body mass index; HCV-RNA, hepatitis C virus ribonucleic acid; SVR 12, sustained viral response at 12 weeks; IFN, interferon; FIB-4, fibrosis-4; AFP, $\alpha$-fetoprotein; DAA, direct-acting antiviral; CTP, Child-Turcotte-Pugh; MELD-NA, model for end-stage liver disease with incorporation of serum sodium; BCLC, Barcelona Clinic Liver Cancer; mUICC, modified Union for International Cancer Control.

${ }^{a}$ Liver transplantation or surgical resection or local ablation or transarterial chomoembolization plus local ablation.

${ }^{\mathrm{b}}$ Transarterial chomoembolization. 
Table 6. Comparison of HCC occurrence in without HCC-history patients

\begin{tabular}{|c|c|c|c|c|c|c|}
\hline \multirow[b]{2}{*}{ Variable } & \multicolumn{3}{|c|}{ Row data } & \multicolumn{3}{|c|}{ Propensity score matching } \\
\hline & $\begin{array}{l}\text { No occurrence } \\
\qquad(n=163)\end{array}$ & $\begin{array}{l}\text { Occurrence } \\
\qquad(\mathrm{n}=5)\end{array}$ & $\begin{array}{c}p \\
\text { value }\end{array}$ & $\begin{array}{l}\text { No occurrence } \\
\qquad(\mathrm{n}=43)\end{array}$ & $\begin{array}{l}\text { Occurrence } \\
\qquad(\mathrm{n}=5)\end{array}$ & $\begin{array}{c}p \\
\text { value }\end{array}$ \\
\hline Age, yr & $57.0(51.0-64.0)$ & $64.0(63.0-79.0)$ & 0.015 & $71.0(63.0-77.0)$ & $64.0(63.0-79.0)$ & 0.879 \\
\hline Female sex & $94(57.7)$ & $1(20.0)$ & 0.168 & $14(32.6)$ & $1(20.0)$ & 0.949 \\
\hline $\mathrm{BMI}$ & $23.3(21.1-25 \cdot 5)$ & $25.9(25.4-28.8)$ & 0.027 & $23.4(21.9-24.9)$ & $25.9(25.4-28.8)$ & 0.009 \\
\hline HCV-RNA & $\begin{array}{c}1,680,000.0 \\
(322,500.0-5,255,000.0)\end{array}$ & $\begin{array}{c}608,000.0 \\
(18,500.0-915,000.0)\end{array}$ & 0.062 & $\begin{array}{c}968,000.0 \\
(138,500.0-5,575,000.0)\end{array}$ & $\begin{array}{c}608,000.0 \\
(18,500.0-915,000.0)\end{array}$ & 0.265 \\
\hline Genotype & & & 1.000 & & & 1.000 \\
\hline 1 & $88(54.0)$ & $3(60.0)$ & & $27(62.8)$ & $3(60.0)$ & \\
\hline 2 & $75(46.0)$ & $2(40.0)$ & & $16(37.2)$ & $2(40.0)$ & \\
\hline DAA for genotype 1 & & & 0.849 & & & 0.676 \\
\hline $\mathrm{DCV}+\mathrm{ASV}$ & $52(59.1)$ & $1(33 \cdot 3)$ & & $15(55 \cdot 6)$ & $1(33 \cdot 3)$ & \\
\hline $\mathrm{EBR}+\mathrm{GZR}$ & $18(20.5)$ & $1(33 \cdot 3)$ & & $7(25 \cdot 9)$ & $1(33 \cdot 3)$ & \\
\hline $\begin{array}{l}\text { OBV/PTV/r + } \\
\text { DSV }\end{array}$ & $10(11.4)$ & $1(33 \cdot 3)$ & & $3(11.1)$ & $1(33 \cdot 3)$ & \\
\hline $\mathrm{SOF}+\mathrm{LDV}$ & $7(7 \cdot 9)$ & o & & $2(7 \cdot 4)$ & o & \\
\hline $\begin{array}{l}\mathrm{SOF}+\mathrm{LDV}+ \\
\mathrm{RBV}\end{array}$ & $1(1.1)$ & ० & & o & o & \\
\hline DAA for genotype 2 & & & 0.945 & & & 1.000 \\
\hline $\begin{array}{l}\mathrm{SOF}+\mathrm{LDV}+ \\
\mathrm{RBV}\end{array}$ & $2(2.7)$ & ० & & $1(6,2)$ & $\mathrm{O}$ & \\
\hline $\mathrm{SOF}+\mathrm{RBV}$ & $71(94.7)$ & $2(100.0)$ & & $15(93.8)$ & $2(100.0)$ & \\
\hline GLE + PIB & $2(2.7)$ & o & & $\mathrm{O}$ & $\mathrm{O}$ & \\
\hline SVR 12 & $160(98.2)$ & $3(60.0)$ & 0.007 & $40(93.0)$ & $3(60.0)$ & 0.130 \\
\hline $\begin{array}{l}\text { Prior IFN } \\
\text { experienced }\end{array}$ & $33(26.0)$ & o & 0.584 & $12(27 \cdot 9)$ & o & 0.413 \\
\hline Liver cirrhosis & $41(26.0)$ & $3(60.0)$ & 0.113 & $15(34 \cdot 9)$ & $3(60.0)$ & 0.542 \\
\hline FIB-4 & $2.5(1.6-3.9)$ & $5 \cdot 9(4.1-6.9)$ & 0.009 & $3.7(2.1-4.7)$ & $5.9(4.1-6.9)$ & 0.094 \\
\hline$>3.25$ & $57(35 \cdot 4)$ & $5(100.0)$ & 0.013 & $24(57 \cdot 1)$ & $5(100.0)$ & 0.169 \\
\hline CTP score & & & 1.000 & & & 1.000 \\
\hline A & $139(94.6)$ & $4(100.0)$ & & $32(86.5)$ & $4(100.0)$ & \\
\hline B & $8(5.4)$ & o & & $5(13 \cdot 5)$ & o & \\
\hline MELD-NA score & & & 1.000 & & & 0.840 \\
\hline$<9$ & $120(73.6)$ & $3(75 \cdot 0)$ & & $22(61.1)$ & $3(75 \cdot 0)$ & \\
\hline $10-19$ & $39(23.9)$ & $1(25.0)$ & & $13(36.1)$ & $1(25.0)$ & \\
\hline $20-29$ & $4(2.5)$ & o & & $1(2.8)$ & 0 & \\
\hline AFP & $4.3(2.8-8.1)$ & $22.9(13.7-28.9)$ & 0.002 & $3.8(2.7-8.6)$ & $22.9(13.7-28.9)$ & 0.006 \\
\hline
\end{tabular}

Values are presented as median (interquartile range) or number (\%).

HCC, hepatocellular carcinoma; BMI, body mass index; HCV-RNA, hepatitis C virus ribonucleic acid; DAA, direct-acting antiviral; DCV, daclatasuvir; ASV, asunaprevir; EBR, elbasvir; GZR, grazoprevir; OBV, ombitasvir; PTV, paritaprevir; r, ritonavir; DSV, dasabuvir; SOF, sofosbuvir; LDV, ledipasvir; RBV, ribavirin; SVR 12, sustained viral response at 12 weeks; IFN, interferon; FIB-4, fibrosis-4; CTP, Child-Turcotte-Pugh; MELD-NA, model for end-stage liver disease with incorporation of serum sodium; AFP, $\alpha$-fetoprotein. 
CHC patients with or without HCC was not significantly different; the HCC recurrence rate was $58.3 \%$ during the median follow-up duration 709.5 days (IQR, 539.0 to 815.5), and the HCC occurrence rate was $3 \%$ during the follow-up of 699.0 days (IQR, 427.5 to 898.5). Although DAA therapy in patients with CHC-related HCC was found to be safe and efficacious, HCC recurrence was relatively common. Therefore, HCC in CHC patients should be considered during treatment decisions, and because of the high risk of recurrence, continuous follow-up is required after treatment.

\section{KEY MESSAGE}

1. This study evaluated the response and safety of direct-acting antiviral (DAA) therapy in chronic hepatitis C (CHC)-related hepatocellular carcinoma (HCC) patients.

2. The study demonstrated that the sustained virologic response at 12 weeks rate of DAA therapy among CHC patients with or without HCC was not significantly different; the cumulative HCC recurrence rate was $58.3 \%$, and the HCC occurrence rate was $3 \%$.

3. Although DAA therapy in CHC-related HCC patients was found to be safe and efficacious, HCC recurrence was common.

\section{Conflict of interest}

No potential conflict of interest relevant to this article was reported.

\section{Acknowledgments}

This work was supported by Konkuk University Medical Center research Grant 2020.

\section{REFERENCES}

1. Polaris Observatory HCV Collaborators. Global prevalence and genotype distribution of hepatitis $\mathrm{C}$ virus infection in 2015: a modelling study. Lancet Gastroenterol Hepatol 2017;2:161-176.

2. El-Serag HB. Epidemiology of viral hepatitis and hepatocellular carcinoma. Gastroenterology 2012;142:1264-1273.
3. Foster GR, Irving WL, Cheung MC, et al. Impact of direct acting antiviral therapy in patients with chronic hepatitis $\mathrm{C}$ and decompensated cirrhosis. J Hepatol 2016;64:12241231.

4. Charlton M, Everson GT, Flamm SL, et al. Ledipasvir and sofosbuvir plus ribavirin for treatment of HCV infection in patients with advanced liver disease. Gastroenterology 2015;149:649-659.

5. Manns M, Samuel D, Gane EJ, et al. Ledipasvir and sofosbuvir plus ribavirin in patients with genotype 1 or 4 hepatitis $\mathrm{C}$ virus infection and advanced liver disease: a multicentre, open-label, randomised, phase 2 trial. Lancet Infect Dis 2016;16:685-697.

6. Huang CF, Yu ML. Direct-acting antivirals response in hepatocellular carcinoma: Does the presence of hepatocellular carcinoma matter? Clin Mol Hepatol 2019;25:168-171.

7. Beste LA, Green PK, Berry K, Kogut MJ, Allison SK, Ioannou GN. Effectiveness of hepatitis C antiviral treatment in a USA cohort of veteran patients with hepatocellular carcinoma. J Hepatol 2017;67:32-39.

8. Shin HR, Kim JY, Kim JI, et al. Hepatitis B and C virus prevalence in a rural area of South Korea: the role of acupuncture. Br J Cancer 2002;87:314-318.

9. Reig M, Marino Z, Perello C, et al. Unexpected high rate of early tumor recurrence in patients with HCV-related HCC undergoing interferon-free therapy. J Hepatol 2016;65:719-726.

10. Conti F, Buonfiglioli F, Scuteri A, et al. Early occurrence and recurrence of hepatocellular carcinoma in HCV-related cirrhosis treated with direct-acting antivirals. J Hepatol 2016;65:727-733.

11. Ide $\mathrm{T}$, Koga $\mathrm{H}$, Nakano $\mathrm{M}$, et al. Direct-acting antiviral agents do not increase the incidence of hepatocellular carcinoma development: a prospective, multicenter study. Hepatol Int 2019;13:293-301.

12. Huang P, Liu M, Zang F, et al. The development of hepatocellular carcinoma in $\mathrm{HCV}$-infected patients treated with DAA: a comprehensive analysis. Carcinogenesis 2018;39:1497-1505.

13. Waziry R, Hajarizadeh B, Grebely J, et al. Hepatocellular carcinoma risk following direct-acting antiviral $\mathrm{HCV}$ therapy: a systematic review, meta-analyses, and meta-regression. J Hepatol 2017;67:1204-1212.

14. European Association for the Study of the Liver; European Organisation for Research and Treatment of Cancer. EASL-EORTC clinical practice guidelines: management 
of hepatocellular carcinoma. J Hepatol 2012;56:908-943.

15. Korean Liver Cancer Association; National Cancer Center. 2018 Korean Liver Cancer Association-National Cancer Center Korea practice guidelines for the management of hepatocellular carcinoma. Gut Liver 2019;13:227-299.

16. Cheung MCM, Walker AJ, Hudson BE, et al. Outcomes after successful direct-acting antiviral therapy for patients with chronic hepatitis $\mathrm{C}$ and decompensated cirrhosis. J Hepatol 2016;65:741-747.

17. Hsu YC, Ho HJ, Wu MS, Lin JT, Wu CY. Postoperative peg-interferon plus ribavirin is associated with reduced recurrence of hepatitis $C$ virus-related hepatocellular carcinoma. Hepatology 2013;58:150-157.

18. Shirabe K, Sugimachi K, Harada N, et al. Favorable prognosis in patients with sustained virological response to antiviral therapy, including interferon, for chronic hepatitis $\mathrm{C}$ before hepatic resection for hepatocellular carcinoma. Anticancer Res 2015;35:6963-6969.

19. Kanogawa N, Ogasawara S, Chiba T, et al. Sustained virologic response achieved after curative treatment of hepatitis C virus-related hepatocellular carcinoma as an independent prognostic factor. J Gastroenterol Hepatol 2015;30:1197-1204.

20. Guarino M, Vigano L, Ponziani FR, et al. Recurrence of hepatocellular carcinoma after direct acting antiviral treatment for hepatitis $\mathrm{C}$ virus infection: literature review and risk analysis. Dig Liver Dis 2018;50:1105-1114.

21. Singh S, Nautiyal A, Loke YK. Oral direct-acting antivirals and the incidence or recurrence of hepatocellular carcinoma: a systematic review and meta-analysis. Frontline Gastroenterol 2018;9:262-270.

22. Yoshimasu Y, Furuichi Y, Kasai Y, et al. Predictive factors for hepatocellular carcinoma occurrence or recurrence after direct-acting antiviral agents in patients with chronic hepatitis C. J Gastrointestin Liver Dis 2019;28:63-71.

23. Pinero F, Mendizabal M, Ridruejo E, et al. Treatment with direct-acting antivirals for HCV decreases but does not eliminate the risk of hepatocellular carcinoma. Liver Int 2019;39:1033-1043.

24. Reig M, Boix L, Marino Z, Torres F, Forns X, Bruix J. Liver cancer emergence associated with antiviral treatment: an immune surveillance failure? Semin Liver Dis 2017;37:109-118.

25. Ohki T, Tateishi R, Sato T, et al. Obesity is an independent risk factor for hepatocellular carcinoma development in chronic hepatitis C patients. Clin Gastroenterol Hepatol 2008;6:459-464. 


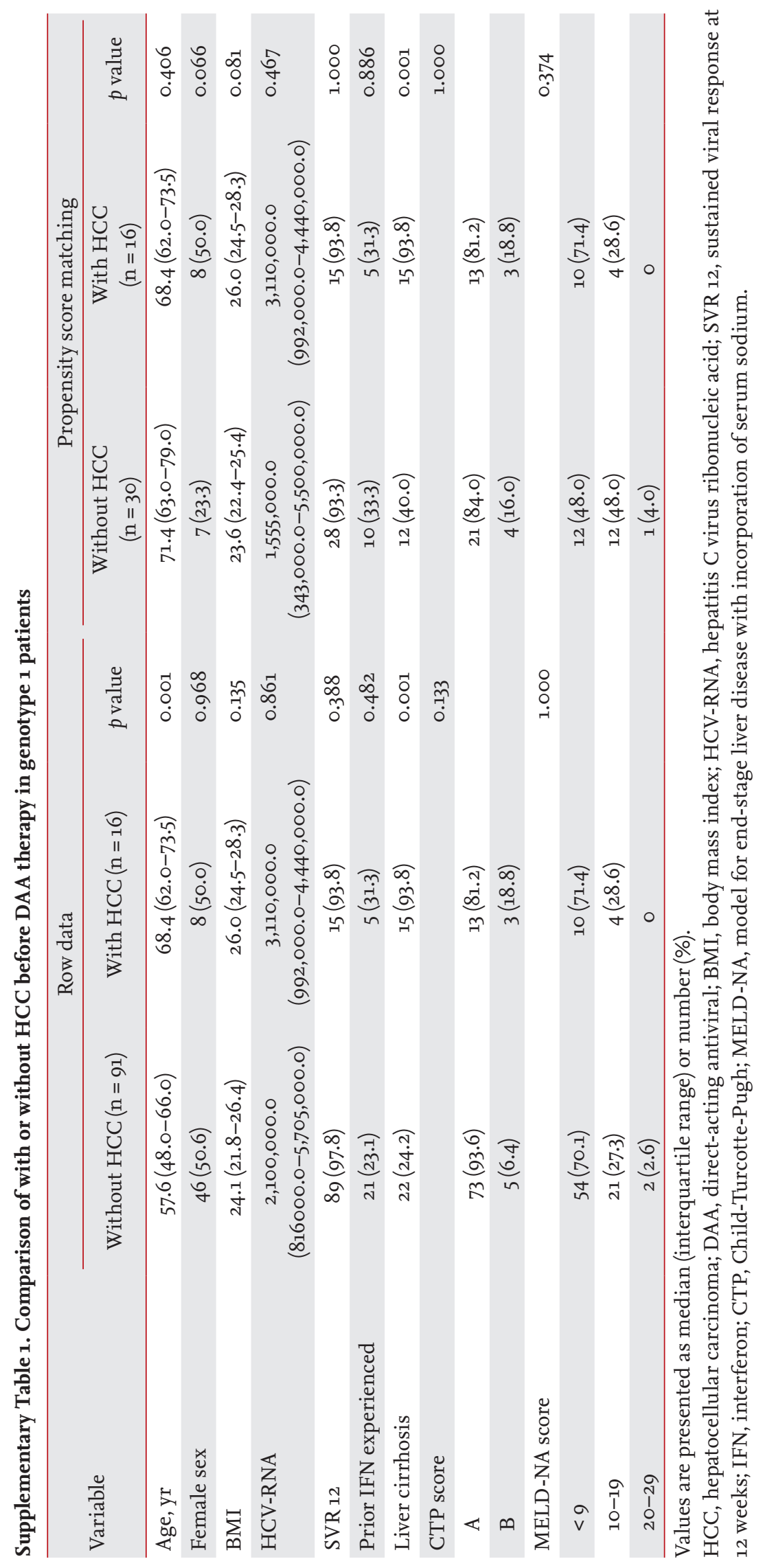




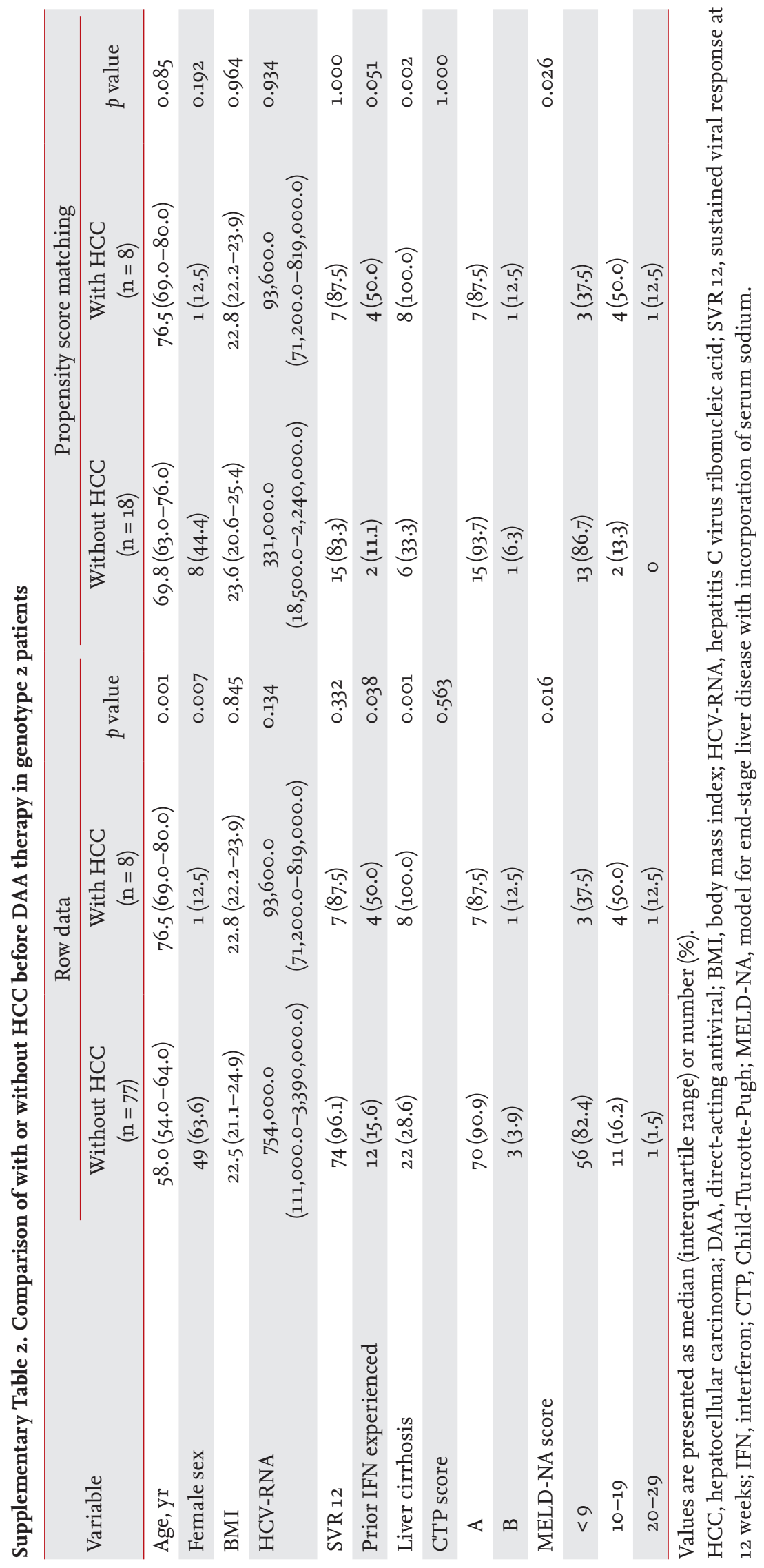

